\title{
Editorial
}

\section{Stoff(e) zum Nachdenken?}

Als ich vor etwas mehr als 20 Jahren für die Abfallwirtschaft begeistert wurde, indem man mir zeigte, dass man die Abfallwirtschaft nicht nur phänomenologisch, sondern vor allem naturwissenschaftlichmethodisch, z. B. mittels Stoffflussanalysen untersuchen muss, war der Fokus der öffentlichen und fachlichen Diskussion sehr auf (Schad-)Stoffe gerichtet. Es waren Fragen wie: Welche Schadstoffgehalte finden wir in den verschiedenen Abfällen und wie verteilen sich diese Schadstoffe bei der Abfallbehandlung auf Deponien, Recyclingprodukte und die Umwelt? Emissionen aus Deponien und Verbrennungsanlagen waren das bestimmende Thema. Seither hat sich viel getan und die Abfallwirtschaft wird heute vornehmlich als Produzent von Sekundärrohstoffen gesehen - von der Abfallwirtschaft zum Ressourcenmanagement. Eigentlich müsste ich froh darüber sein. Endlich weg von der früheren einseitigen Schadstoffsicht hin zum Versuch einer umfassenden Bewirtschaftung der Abfälle bzw. des gesamten volkswirtschaftlichen Güter- und Stoffhaushaltes. Zumindest in ersten Zügen. Trotzdem habe ich ein ungutes Gefühl bei dieser Entwicklung. Warum?

An unserem Institut führen wir eine Laborübung durch. Sie soll den Studierenden einen Einblick geben, welcher analytische Aufwand zu treiben ist, um Produkte oder Abfälle auf ihre Schadstoffgehalte zu untersuchen. Die Untersuchungen beziehen sich dabei im Wesentlichen auf die üblichen Verdächtigen aus der Gruppe der Schwermetalle. Damit die Übung auch für unser Laborpersonal interessant bleibt, werden jedes Jahr andere Produkte bzw. Abfälle beprobt. Es kann also sein, dass wir die Studierenden in den Baumarkt schicken, um Komposterde, Spanplatten, Energiesparlampen, Batterien oder Zement zu kaufen. Eine Eigenheit, ich würde sagen Stärke, unseres Laborteams ist es, dass die Proben nicht stur nach Norm aufbereitet und analysiert werden, sondern je nach Produkt zusätzlich ein Vorgehen gewählt wird, das uns hoffen lässt, den wahren Stoffgehalten möglichst nahe zu kommen. Also Analyse mit Herz und Hirn.

Und was kommt dabei heraus? Nicht immer das, was wir erwarten. Zum Beispiel Komposte, welche die Grenzwerte der angegebenen Qualitätsklasse teils deutlich überschreiten. Oder Zemente, in denen der Quecksilbergehalt immer unterhalb der Nachweisgrenze (nicht Bestimmungsgrenze) liegt. Aber müssten diese Gehalte nicht höher sein, wenn wir uns die Quecksilberbilanz der Zementindustrie vor Augen halten? In Rohmaterialien und Brennstoffen ist Quecksilber enthalten und wenn es nicht im Zement zu finden ist, wo ist es dann? Natürlich sind das nur Gedanken und es ist völlig klar, dass wir hier keine repräsentativen Untersuchungen vorliegen haben. Eine Laborübung kann das nicht. Wahrscheinlich ist auch alles in Ordnung, aber weiß man's? Muss man da nicht genauer hinsehen?

Wie etwa bei den Ersatzbrennstoffen, wo wir dies zur Abklärung der Machbarkeit von Phosphorrecycling-Projekten tun mussten. Dort fällt auf, dass die Analyse nach Norm häufig Minderbefunde ergibt und die Angaben der Ersatzbrennstoffanbieter mit den analysierten Werten nicht übereinstimmen. Sind das überhaupt Ersatzbrennstoffe, wenn die Schwermetallgehalte im Bereich von Restmüll und nicht von zumindest Steinkohle liegen? Ersatzbrennstoffe ersetzen ja fossile Brennstoffe und nicht Restmüll.

Ich vermisse eine stoffliche und bilanzielle Betrachtung der Abfall- und Recyclingwirtschaft. Eine solche ist nicht einfach zu realisieren, aber sie ist notwendig und möglich. Sie ist notwendig, weil man damit Analysenergebnisse kontrollieren kann und ein systemisches Verständnis über Quantität und Qualität der Abfälle, Recyclingprodukte und Emissionen erhält. Sie ist auch möglich, da wir heute im 


\section{Editorial}

Vergleich zu vor 20 Jahren über wesentlich bessere Voraussetzungen und Instrumente verfügen. Es ist mittlerweile mehr eine Frage des Wollens als des Könnens. Aber vielleicht ist mein Unbehagen unbegründet. Zu hoffen wär's.

Univ.-Prof. DI Dr. H. Rechberger ( $ه$ )

Institut für Wassergüte, Ressourcen-

management und Abfallwirtschaft,

Technische Universität Wien,

Karlsplatz 13/226,

1040 Wien, Österreich

helmut.rechberger@tuwien.ac.at 